This item was submitted to Loughborough's Research Repository by the author.

Items in Figshare are protected by copyright, with all rights reserved, unless otherwise indicated.

\title{
Asymmetric long Josephson junction acting as a ratchet for a quantum field
}

PLEASE CITE THE PUBLISHED VERSION

http://dx.doi.org/10.1103/PhysRevLett.104.190602

\section{PUBLISHER}

(C) American Physical Society

VERSION

VoR (Version of Record)

LICENCE

CC BY-NC-ND 4.0

REPOSITORY RECORD

Sboychakov, A.O., Sergey Savel'ev, Alexander L. Rakhmanov, and Franco Nori. 2019. "Asymmetric Long Josephson Junction Acting as a Ratchet for a Quantum Field”. figshare. https://hdl.handle.net/2134/12722. 
This item was submitted to Loughborough's Institutional Repository (https://dspace.lboro.ac.uk/) by the author and is made available under the following Creative Commons Licence conditions.

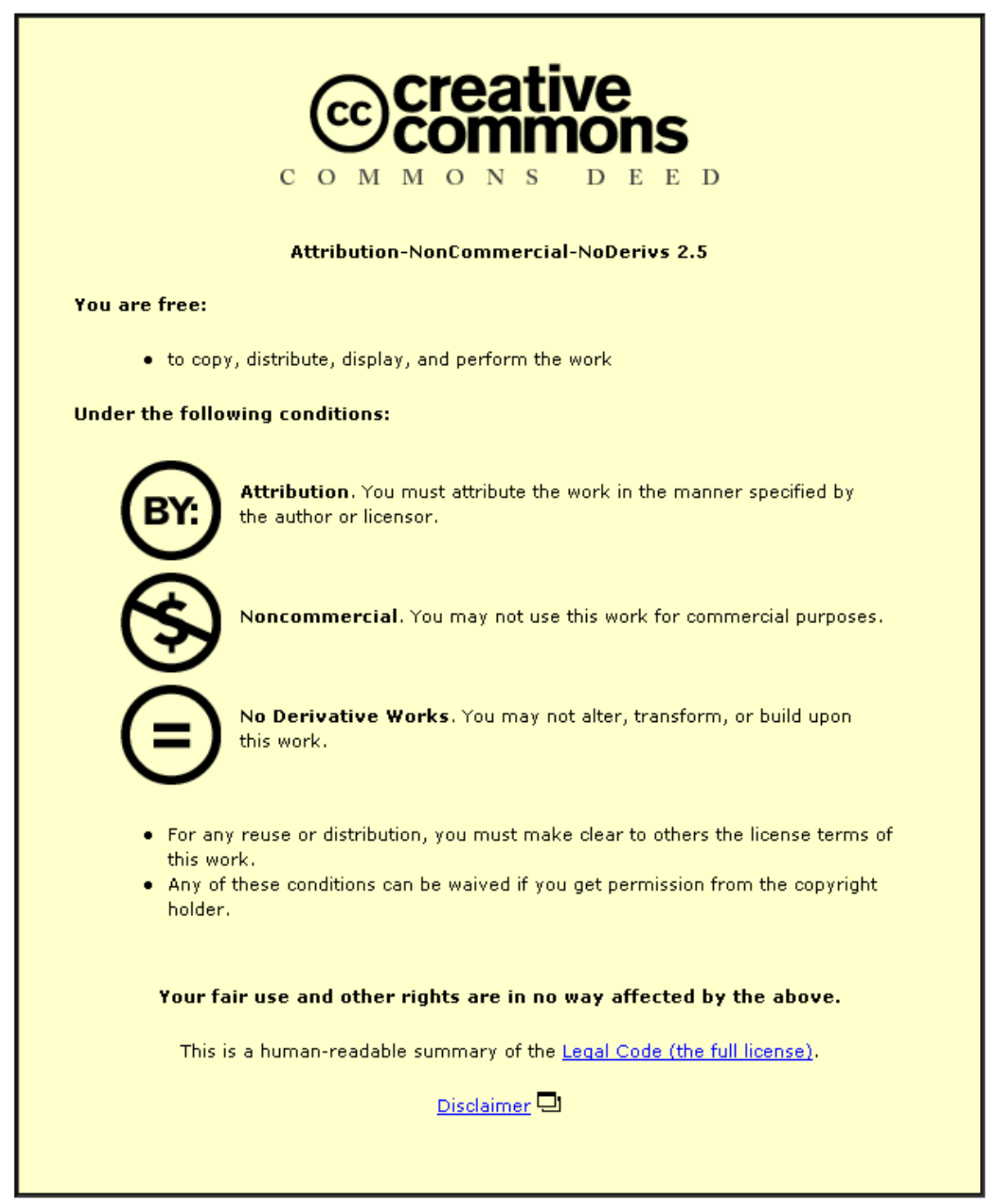

For the full text of this licence, please go to: http://creativecommons.org/licenses/by-nc-nd/2.5/ 


\title{
Asymmetric Long Josephson Junction Acting as a Ratchet for a Quantum Field
}

\author{
A. O. Sboychakov, ${ }^{1,2}$ Sergey Savel'ev, ${ }^{1,3}$ A. L. Rakhmanov, ${ }^{1,2}$ and Franco Nori ${ }^{1,4}$ \\ ${ }^{1}$ Advanced Science Institute, RIKEN, Wako-shi, Saitama, 351-0198, Japan \\ ${ }^{2}$ Institute for Theoretical and Applied Electrodynamics Russian Academy of Sciences, 125412 Moscow, Russia \\ ${ }^{3}$ Department of Physics, Loughborough University, Loughborough LE11 3TU, United Kingdom \\ ${ }^{4}$ Department of Physics, The University of Michigan, Ann Arbor, Michigan 48109-1040, USA
}

(Received 10 August 2009; published 13 May 2010)

\begin{abstract}
We study the escape rate of flux quanta in a long Josephson junction having an asymmetric spatial inhomogeneous critical current density. Such a junction can behave as a ratchet when driven by an ac current in the presence of a magnetic field. This rectification gives rise to a dc voltage $V_{\mathrm{dc}}$ across the junction. The usual approach of particlelike tunneling cannot describe this rectification, and a quantum field theory description is required. We also show that, under specific conditions, the rectification direction, and consequently $V_{\mathrm{dc}}$, can change its sign when varying the temperature $T$ near the crossover temperature $T^{*}$ between the quantum and classical regimes.
\end{abstract}

DOI: 10.1103/PhysRevLett.104.190602

PACS numbers: 05.40.- a, 74.50.+r, 85.25.Cp

Introduction.-Solid state devices with asymmetric periodic potentials (acting as ratchets) are attracting considerable interest [1]. Ratchets can produce a direct current when driven by nonequilibrium noise. Moreover, for some special shapes of the ratchet potential, the current can change its sign for decreasing temperatures, when quantum tunneling becomes dominant over thermal-activated hopping [2]. There are many different realizations of ratchets, both in nature and in artificial nanodevices, such as cold atoms, colloidal magnetic particles, single-molecule optomechanical devices, fluxons in superconductors, and many other systems (for reviews, see, e.g., Ref. [1]). All these systems are effectively described by a quantum or classical particle moving in a periodic asymmetric potential.

Here we propose a completely new ratchet system, which is described by a quantum field. Namely, we consider a long Josephson junction (JJ) (the junction's length $D$ is comparable with the Josephson penetration depth $\lambda_{J}$ ) with a spatially modulated critical current density $i_{c}(x)$ driven by nonequilibrium fluctuations sustained by an ac current $J=J_{0} \cos \Omega t$, where the amplitude $J_{0}$ is right below the junction's critical current $J_{c}$. The gaugeinvariant phase difference $\varphi(x)$ plays the role of the field variable. Although $J$ never exceeds $J_{c}$, the flowing current can give rise to sudden changes in the phase difference, both due to quantum tunneling through, and thermal hopping over the potential barrier. The probability per unit time (= escape rate) $\Gamma(J)$ of these events attains maxima $\Gamma_{ \pm}$at $J= \pm J_{0}$, and one can expect that $\Gamma_{+}=\Gamma_{-}$. However, the application of an external dc magnetic field $H$ to the junction having a current inhomogeneity asymmetric with respect to the $x$ direction results in the asymmetry of both the tunneling probability, $\Gamma_{+}(I) \neq \Gamma_{-}(-I)$, and the critical currents, $J_{c}^{+} \neq J_{c}^{-}$, for applied currents flowing in opposite directions. Namely, both $\Gamma$ and $J_{c}$ depend on the direction of the applied current. This leads to the appearance of a dc voltage $V_{\mathrm{dc}}$ across the junction, since $V_{\mathrm{dc}}=(\hbar / 2 e)\left\langle\frac{\partial \varphi}{\partial t}\right\rangle \propto\left(\Gamma_{+}-\Gamma_{-}\right)$. In the region where noise is classical, $\hbar \omega_{p} \ll T$ (here $\omega_{p}$ is the plasma frequency), the escape rates $\Gamma_{ \pm}$are determined by thermal hopping, and only the height $\Delta U$ of the potential barrier is important. Since, in the classical regime, $\Delta U$ is proportional to the critical currents $J_{c}^{ \pm}$, one can easily predict the net voltage (rectification) sign if the values of $J_{c}^{ \pm}$are known. However, when quantum noise starts to play a crucial role, $\hbar \omega_{p} \gtrsim T$, escape rates $\Gamma_{ \pm}$are governed by both barrier height and its width. Thus, we could expect a counterintuitive current inversion, when $\Gamma_{+}<\Gamma_{-}$even though $J_{c}^{+}>J_{c}^{-}$. A main result of this work is the demonstration that this effect can be observed for asymmetric JJs. The particlelike approach used in most theoretical considerations of quantum tunneling in JJs is not appropriate here, and a field-theoretical description must be used.

The effect of quantum tunneling in JJs and stacks of intrinsic JJs in high- $T_{c}$ superconductors has been studied intensively, experimentally [3], and theoretically [4], both due to its fundamental interest and the possibility to use these systems in future applications. A ratchet based on a SQUID consisting of two equal JJs in series, coupled in parallel to a third junction, was proposed in Ref. [5]. Also a remarkable Josephson vortex ratchet in a long JJ was studied in Ref. [6].

We stress that the system under consideration here does not correspond to a ratchet in the usual sense, because the junction's potential is the periodic sine-Gordon potential, $U(\varphi) \propto-\cos \varphi$, which is symmetric in $\varphi$ (another example of a ratchet with symmetric potential was proposed in Ref. [7]). The difference between $\Gamma_{+}$and $\Gamma_{-}$(and, consequently, a voltage rectification) occurs here due to both: the "parametric" dependence of the potential $U$ on $x$, and the spatial dependence of the field $\varphi(x)$. Thus, the field character of the phase difference $\varphi(x)$ is crucial for the 
rectification, even for relatively short junctions. We believe that the developed theory can be applied not only to JJs, but to other distributed systems, where a field description is required, e.g., nanobars, magnetic vortices, stacks of intrinsic high- $T_{c}$ JJs, etc. Here we exploit the approach developed in Ref. [8], which we generalize to finite temperatures. This now allows us to calculate, in the same manner, the escape rate $\Gamma=A \exp (-B)$ both in the quantum and classical regimes. If the junction length $D$ is large, then the exponent $B$ of the escape rate $\Gamma$ turns out also to be large. However it should be not too large, when $\Gamma$ becomes an experimentally nonobservable quantity. This last condition means that the current amplitude $J_{0}$ should be close to the critical current. We focus on the exponent $B$ because changes in $B$ (not in the prefactor $A$ ) describe the main change in $\Gamma$ when $B$ is large. In addition, here we do not consider dissipation in the system when calculating $B$. Dissipation does not qualitatively change our results.

Escape rate.-We consider a Josephson junction in the inline geometry shown in the inset of Fig. 1. Two superconducting bars overlap a length $D$ in the $x$ direction. The external magnetic field $H$ is applied in the $y$ direction. Let us first consider the junction biased by a dc current $J$. The generalization to the adiabatically varying ac current $J \cos \Omega t$ is evident, and the conditions of adiabaticity will be given below. The spatial inhomogeneity of the critical current density $i_{c}(x)$ in the $x$ direction can be realized, e.g., by changing the shape of the junction [6]. We calculate the escape rate $\Gamma=A \exp (-B)$ using the well-known method of imaginary-time trajectories at finite temperatures [9], where the prefactor $A \sim \omega_{p}$, and $B=S_{\beta} / \hbar$. Here, $S_{\beta}$ is the action of the system for a periodic imaginary-time trajectory, $\varphi(\tau+\hbar \beta)=\varphi(\tau)$, with a period $\tau_{0}=\hbar \beta$, $\beta=1 / T$. The quasiclassical imaginary-time action is

$$
\begin{aligned}
\mathcal{S}_{\beta}[\varphi] & =\frac{E_{J}}{\omega_{p}} \int_{0}^{\hbar \omega_{p} \beta} d \tau\left\{\mathcal{L}[\varphi]+\mathcal{L}_{\Sigma}[\varphi]\right\}, \\
\mathcal{L}[\varphi] & =\int_{-d / 2}^{d / 2} d x\left[\frac{1}{2}\left(\frac{\partial \varphi}{\partial \tau}\right)^{2}+\frac{1}{2}\left(\frac{\partial \varphi}{\partial x}\right)^{2}-g(x) \cos \varphi\right], \\
\mathcal{L}_{\Sigma}[\varphi] & =\varphi\left(\tau,-\frac{d}{2}\right)\left[h-\frac{I}{2}\right]-\varphi\left(\tau, \frac{d}{2}\right)\left[h+\frac{I}{2}\right] .
\end{aligned}
$$

In Eqs. (1), the $x$ coordinate is normalized by $\lambda_{J}, \tau$ by $1 / \omega_{p}, d=D / \lambda_{J}, E_{J}=\hbar \overline{i_{c}} \lambda_{J} L /(2 e)$ is the Josephson energy ( $L$ is the junction's width in the $y$ direction), the bar above $i_{c}$ means spatial averaging, and $g(x)=i_{c}(x) / \overline{i_{c}} \equiv$ $1+\gamma(x), \overline{\gamma(x)}=0$. The phase $\varphi(\tau, x)$ satisfies the equation of motion

$$
\frac{\partial^{2} \varphi}{\partial \tau^{2}}+\frac{\partial^{2} \varphi}{\partial x^{2}}-g(x) \sin \varphi=0,
$$

with the periodicity condition $\varphi\left(\tau+\hbar \omega_{p} \beta, x\right)=\varphi(\tau, x)$. The boundary conditions for the phase difference $\varphi(\tau, x)$ are defined by the $\mathcal{L}_{\Sigma}$ term in the action (1): $\partial \varphi /\left.\partial x\right|_{x= \pm d / 2}= \pm I / 2+h$, where $I$ and $h$ are the dimen-

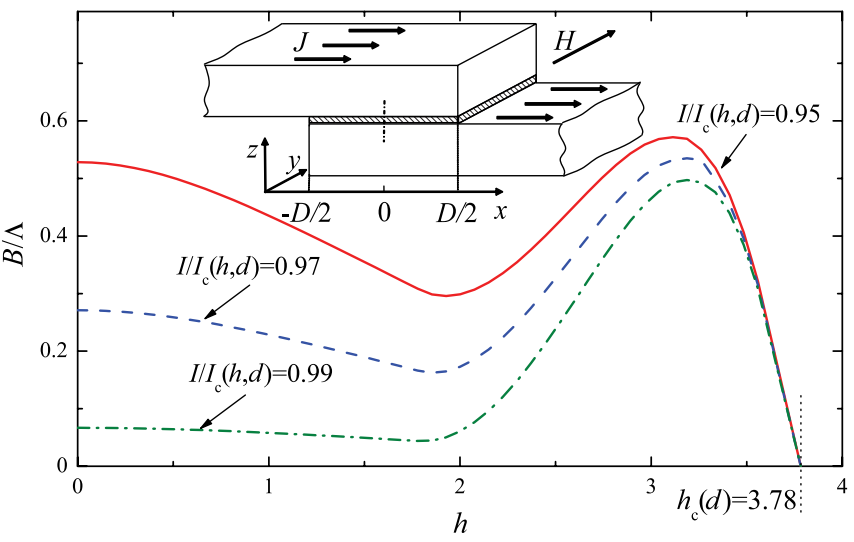

FIG. 1 (color online). The magnetic field dependence of the escape rate's exponent $B$ for fixed current ratios $I / I_{c}(h, d)$ in the uniform junction $[\gamma(x)=0]$, calculated according to Eq. (6); $d=1.8$ and $T=0$. The inset shows the schematic geometry of the Josephson junction.

sionless current and external magnetic field, respectively: $I=J /\left(\overline{i_{c}} \lambda_{J} L\right)$, and $h=c H /\left(4 \pi \overline{i_{c}} \lambda_{J}\right)$.

In general, it is hard to find a periodic solution to the nonlinear Eq. (2), even numerically. However, as mentioned above, we only need to find a solution when the current $I \approx I_{c}$. In this case, we can use the approach developed in Ref. [8]. Namely, we seek a solution of the form $\varphi(\tau, x)=\varphi_{0}(x)+\psi(\tau, x)$, where $\varphi_{0}(x)$ is the steady-state solution of Eq. (2) corresponding to an energy minimum. Since $I$ is close to the critical current, the energy barrier between the neighboring energy minima, $\varphi_{0}(x)$ and $\varphi_{0}(x) \pm 2 \pi$, is small, $|\psi(x, \tau)| \ll \varphi_{0}(x)$, and we can expand the action (1) in powers of $\psi$ up to $\psi^{3}$. Then, we represent $\psi(\tau, x)$ in the form of a series

$$
\psi(\tau, x)=\frac{3 \mu_{0}}{U_{000}} \sum_{n=0}^{\infty} \alpha_{n}\left(\sqrt{\mu_{0}} \tau\right) \psi_{n}(x)
$$

where $\mu_{n}$ and $\psi_{n}(x)$ are the eigenvalues and orthogonal eigenfunctions of the operator $\hat{\mathcal{D}}=-\partial^{2} / \partial x^{2}+g(x) \times$ $\cos \varphi_{0}(x), \hat{\mathcal{D}} \psi_{n}=\mu_{n} \psi_{n}$, and $U_{n m k}=\int_{-d / 2}^{d / 2} d x g(x) \times$ $\sin \varphi_{0} \psi_{n} \psi_{m} \psi_{k}$.

Expanding the equation of motion (2) in powers of $\psi$, up to $\psi^{2}$, with $\psi$ in the form (3), multiplying it by $\psi_{n}(x)$, and integrating over $x$, we obtain an infinite system of ordinary differential equations for the collective coordinates $\alpha_{n}(\eta)$ of the field $\psi$, where we introduce the imaginary-time variable $\eta=\sqrt{\mu_{0}} \tau$. It can be shown that due to the proximity of $I$ to $I_{c}$, two conditions for the eigenvalues $\mu_{n}$ are possible: either $\mu_{0} \ll \mu_{n}, n>0$ for relatively short junctions $(d \lesssim 1)$ or large fields $(h \gtrsim 1)$; or $\mu_{0} \sim \mu_{1} \ll \mu_{n}$, $n>1$ for long junctions $(d \gtrless 1)$ and small fields $(h \ll 1)$. Because of these inequalities, one can neglect all the equations in the system of equations for $\alpha_{n}$, except the first two (for details, see Ref. [8]). Thus, the system of equations for $\alpha_{0}$ and $\alpha_{1}$ takes the form 


$$
\ddot{\alpha}_{0}+\frac{\partial V}{\partial \alpha_{0}}=0, \quad \ddot{\alpha}_{1}+\frac{\partial V}{\partial \alpha_{1}}=0,
$$

where the dot means derivative over $\eta$, and the potential $V\left(\alpha_{0}, \alpha_{1}\right)$ can be written as

$$
V=\frac{1}{2} \sum_{i=0,1}\left[\frac{U_{i i i} \alpha_{i}^{3}+3 U_{01 i} \alpha_{0} \alpha_{1} \alpha_{i}}{U_{000}}-\frac{\mu_{i} \alpha_{i}^{2}}{\mu_{0}}\right] .
$$

We should find a periodic solution of the system (4) with period $\eta_{0}=\sqrt{\mu_{0}} \hbar \omega_{p} / T$ ("bounce" solutions). The exponent $B=S_{\beta} / \hbar$ of the escape rate $\Gamma=A \exp (-B)$ can be expressed through the functions $\alpha_{i}(\eta), i=0,1$ as

$$
\begin{aligned}
& B=\frac{9 \Lambda \mu_{0}^{5 / 2}}{\left(U_{000}\right)^{2}} \int_{0}^{\eta_{0}} d \eta\left[\sum_{i} \frac{\dot{\alpha}_{i}^{2}}{2}-V\left(\alpha_{0}, \alpha_{1}\right)\right], \\
& \Lambda=\frac{E_{J}}{\hbar \omega_{p}} .
\end{aligned}
$$

Thus, we reduce the field-theory problem to the problem of one particle moving in the effective potential $V\left(\alpha_{0}, \alpha_{1}\right)$ in two dimensions (not in 1D as in the usual particlelike approximation), where the $\alpha_{i}$ 's play the role of the particle's generalized coordinates. Let us first consider the case of $T \rightarrow 0$, when quantum tunneling prevails. Our analysis shows that when $d>d_{c}(I, h) \sim 4$, there exist three bounce solutions to the system (4): $\alpha_{i}^{(s)}(\eta), s=0, \pm 1$. In analogy to the case $\gamma(x)=h=0$ considered in Ref. [8], one can say that the solution $\alpha_{i}^{(+1)}(\eta)\left(\alpha_{i}^{(-1)}(\eta)\right)$ corresponds to the formation of a vortex (antivortex) nucleus at the left (right) junction edge, while the solution $\alpha_{i}^{(0)}(\eta)$ describes the tunneling of $\varphi$ as a whole (for details, see Ref. [8]). When $\gamma(x)=h=0$, the solutions have the following symmetric properties: $\alpha_{1}^{(0)}(\eta)=0, \alpha_{1}^{(-1)}(\eta)=-\alpha_{1}^{(+1)}(\eta)$. Thus we have three channels of tunneling, with probabilities $\Gamma^{(s)} \propto$ $\exp \left(-B^{(s)}\right)$, with $B^{(-1)}=B^{(+1)}$, and the total probability becomes $\Gamma=\sum_{s} \Gamma^{(s)}$. The applied magnetic field breaks the vortex-antivortex symmetry $\left[B^{(-1)}=B^{(+1)}\right]$ making one of these channels more favorable. However, the total escape rate $\Gamma$ is still symmetric with respect to the direction of the dc current if $\gamma(x)=0$. For spatially inhomogeneous junctions with $\gamma(x) \neq 0$, we have $\Gamma(+I) \neq \Gamma(-I)$, and the rectification $V_{\mathrm{DC}}=\frac{1}{2}\{V(+I)+V(-I)\} \propto\left(\Gamma_{+}-\Gamma_{-}\right)$ arises. Since the main contribution to the total $\Gamma$ comes from the term corresponding to the minimum of $B^{(s)}$, we will assume below that $\Gamma \propto \exp (-B)$, where $B=$ $\min \left(B^{(s)}\right)$. When $d<d_{c}(I, h)$ there is only one solution to the system (4), $\alpha_{i}^{(0)}(\eta)$. When $h \neq 0$ and/or $\gamma(x) \neq 0$, we have $\alpha_{1}^{(0)} \neq 0$, and, in contrast to the case studied in Ref. [8], a 2D consideration is required here, because there is no ratchet effect in a point (i.e., no $x$ dependence) Josephson junction model (i.e., for a 1D particle approximation).
All these properties of the bounce solutions $\alpha_{i}^{(s)}$ survive at finite temperatures up to some value $T^{*}$, which is the crossover temperature between the quantum and classical regimes. At low temperatures, $T<T^{*}$, the exponent $B$ only slightly decreases with $T$ (thermally stimulated tunneling). When $T>T^{*}$, there are only imaginary-time independent solutions to Eq. (4), $\alpha_{i}^{(s)}(\eta)=\bar{\alpha}_{i}^{(s)}$, where the points $\left(\bar{\alpha}_{0}^{(s)}\right.$, $\left.\bar{\alpha}_{1}^{(s)}\right)$ correspond to the extremes (minimum or saddlepoints) of the potential $V\left(\alpha_{0}, \alpha_{1}\right)$ (here $s$ takes the values $s=0, \pm 1$, if $d>d_{c}(I, h)$, and $s=0$ otherwise). Thus, when $T>T^{*}$ the exponent $B$ can be written as

$$
B=\frac{9 \Lambda \mu_{0}^{3}}{\left(U_{000}\right)^{2}} \frac{\hbar \omega_{p}\left|V_{r}\right|}{T}, \quad V_{r}=\max _{s}\left[V\left(\bar{\alpha}_{0}^{(s)}, \bar{\alpha}_{1}^{(s)}\right)\right]
$$

The crossover temperature $T^{*}$ is the temperature where the period of the bounce solution, $\eta_{0}=\sqrt{\mu_{0}} \hbar \omega_{p} / T$, becomes equal to the period of infinitesimal oscillations near the extreme point $\left(\bar{\alpha}_{0}^{(r)}, \bar{\alpha}_{1}^{(r)}\right)$, corresponding to the maximum of $V\left(\bar{\alpha}_{0}^{(s)}, \bar{\alpha}_{1}^{(s)}\right)$. The latter one is equal to $2 \pi / \sqrt{\lambda_{r}^{+}}$, where $\lambda_{r}^{+}$is the positive eigenvalue of the matrix $\partial^{2} V / \partial \alpha_{i} \partial \alpha_{j}$ calculated at the point $\left(\bar{\alpha}_{0}^{(r)}, \bar{\alpha}_{1}^{(r)}\right)$. As a result, the crossover temperature can be written as $T^{*}=\hbar \omega_{p} \sqrt{\mu_{0} \lambda_{r}^{+}} /(2 \pi)$.

All results above were obtained for a dc current. We can also use all the above formulas for a slowly-varying current $I=I_{0} \cos \Omega t$, assuming that at any time $t$ the current can be considered as a dc one. This adiabatic limit is valid when the frequency $\Omega$ is much smaller than the inverse imaginary-time period, $1 / \tau_{0}=T / \hbar$. Therefore, we obtain the condition of adiabaticity [10]: $\hbar \Omega \ll T$.

Results and discussions. - Let us first neglect the spatial distribution of the critical current density, assuming that $\gamma(x)=0$. In this case, the following condition is met $B(-I, h)=B(I, h)$, and there is no rectification. In Fig. 1, we plot the magnetic field dependence of the exponent $B(T=0)$ for three fixed ratios $I / I_{c}(h, d)$, where the critical current $I_{c}(h, d)$ depends on the magnetic field. It goes to zero when $h$ achieves the critical field $h_{c}(d)$. Note the nonmonotonic dependence of $B$ on $h$, which is related to the change in characteristic properties of the static solution $\varphi_{0}(x)$ with growing $h$. The nonzero $\gamma(x)$ breaks down the symmetry of $B$ with respect to the direction of the current. In this case, we have two critical currents $I_{c}^{ \pm}$, corresponding to positive and negative directions of the current. Figure 2 shows the dependence of $B( \pm I, h)$ and $\left(1-I_{c}^{-} / I_{c}^{+}\right)$on $h$, calculated for a cubic-minus-linear $\gamma(x)$ at the fixed ratio $I / I_{c}(h, d)$, where $I_{c}(h, d)$ is the critical current calculated for $\gamma=0$. The ratio $I_{c}^{-} / I_{c}^{+}<1$ for any value of the magnetic field, while $B_{-}>B_{+}$in some region of $h$ and $B_{-}<B_{+}$in other regions; thus, resulting in a net voltage inversion. This reflects the fact that the probability of quantum tunneling is determined not only by the barrier's height, but also by the barrier's width. In our case, the former one is determined mainly by the critical currents 


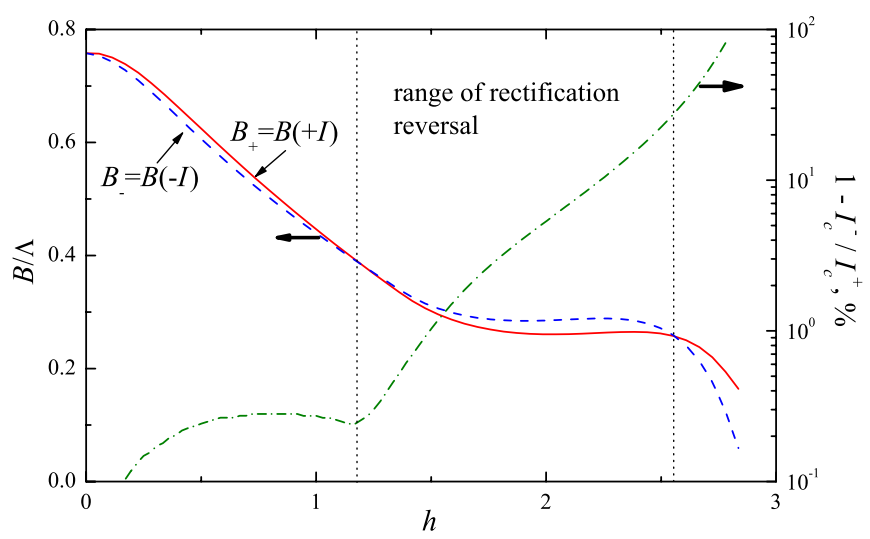

FIG. 2 (color online). The magnetic field dependence of $B_{+}=$ $B(+I, h)$ (red solid curve) and $B_{-}=B(-I, h)$ (blue dashed curve) for a fixed ratio $I / I_{c}=0.95$, calculated for $\gamma(x)=$ $0.27 x^{3}-0.17 x$. The relative ratio $\left(1-I_{c}^{-} / I_{c}^{+}\right)$as a function of $h$ is shown by the green dotted-dashed curve. Here the parameters are $d=2.5, T=0,\left(\overline{\gamma^{2}(x)}\right)^{1 / 2}=0.1$. In the region of $h$ separated by the vertical dotted lines, we obtain $B_{-}>B_{+}$ although $I_{c}^{-}<I_{c}^{+}$. For $h$ in this region, the change of sign of the rectification takes place when increasing $T$.

$I_{c}^{ \pm}$, while the last one substantially depends on the $\gamma(x)$ profile.

Let us now consider the temperature dependence of the escape rate exponent $B$, going from classical hopping (at high $T$ ) to quantum tunneling (at low $T$ ). The curves $B_{ \pm}=$ $B( \pm I)$ versus $T$ are shown in Fig. 3. The difference $\left(B_{+}-\right.$ $\left.B_{-}\right)$, which defines a ratchet effect as a function of temperature, is also shown in Fig. 3. It changes sign for temperatures near the crossover temperature between the quantum and classical regimes. The sign of the difference $\left(B_{+}-B_{-}\right)$defines the sign of the rectified voltage $V_{\mathrm{dc}}$. Thus, the effect of the change of sign of $\left(B_{+}-B_{-}\right)$can be observed by measuring the dc voltage as a function of temperature. The value $\left(B_{+}-B_{-}\right)$can be calculated using data of the escape rate $\Gamma$ obtained either in ac or dc current measurements.

Conclusions.-We have proposed a new type of ratchet system where the dynamical variable is a field. Namely, we have considered a long $\mathrm{JJ}$ with a spatially inhomogeneous critical current density $i_{c}(x)$. In this case, the field character of the phase difference is crucial for the ratchet effect. The exponent of the escape rate of the phase difference $\varphi$ was calculated as a function of: temperature, dc magnetic field, and dc or slow ac current. We have shown that due to both the magnetic field and the spatial inhomogeneity of $i_{c}$, the escape rate becomes asymmetric with respect to the direction of the current. This leads, in particular, to the appearance of a dc voltage when the system is biased by an ac current. We have also shown that, for definite shapes of $i_{c}(x)$, the rectified voltage changes sign for $T$ near the crossover temperature $T_{*}$ between the quantum-tunneling and classical-hopping regimes. Thus, the proposed ratchet

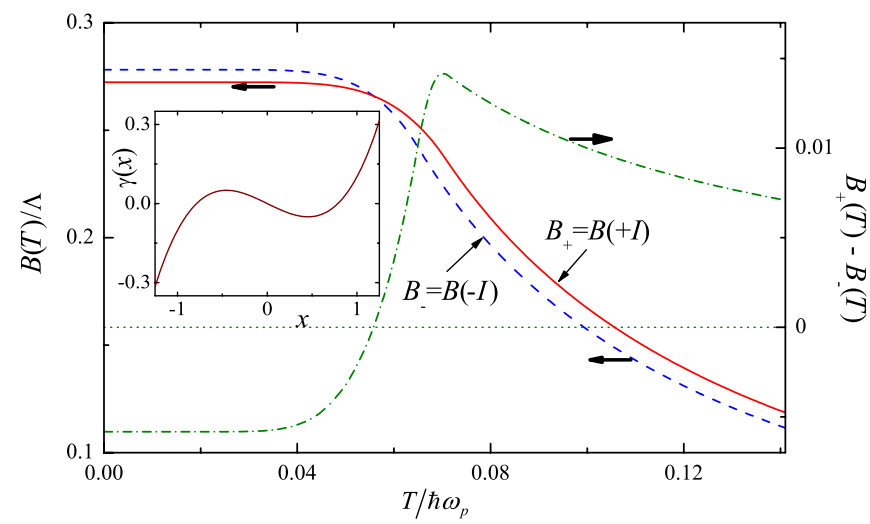

FIG. 3 (color online). The escape rate exponents $B(+I)$ (red solid curve) and $B(-I)$ (blue dashed curve) versus temperature calculated for $\gamma(x)=0.27 x^{3}-0.17 x$. The difference $B(+I)-$ $B(-I)$ is shown by the green dotted-dashed curve. The other parameters are: $d=2.5, h=2.25, I / I_{c}=0.95$. The profile $\gamma(x)$ is shown in the inset.

system demonstrates that the net voltage can change sign when $T$ is lowered for the same external forces when quantum fluctuations start to dominate.

This work was supported in part by the RFBR 09-0200248, JSPS-RFBR 09-02-92114, the US NSA, LPS, ARO, and NSF. SS acknowledges support from the EPSRC via EP/D072581/1 and EP/F00548211. A. O.S. acknowledges support from the RSSF.

[1] P. Hänggi, F. Marchesoni, and F. Nori, Ann. Phys. (Leipzig) 14, 51 (2005); P. Hänggi and F. Marchesoni, Rev. Mod. Phys. 81, 387 (2009).

[2] P. Reimann, M. Grifoni, and P. Hänggi, Phys. Rev. Lett. 79, 10 (1997).

[3] K. Inomata et al., Phys. Rev. Lett. 95, 107005 (2005); X. Y. Jin et al., ibid. 96, 177003 (2006); T. Bauch et al., Science 311, 57 (2006).

[4] S. Kawabata et al., Phys. Rev. B 76, 064505 (2007); M. Machida and T. Koyama, Supercond. Sci. Technol. 20, S23 (2007).

[5] I. Zapata et al., Phys. Rev. Lett. 77, 2292 (1996).

[6] E. Goldobin, A. Sterch, and D. Koelle, Phys. Rev. E 63, 031111 (2001); M. Beck et al., Phys. Rev. Lett. 95, 090603 (2005).

[7] M. Buttiker, Z. Phys. B 68, 161 (1987).

[8] S. Savel'ev, A. L. Rakhmanov, and F. Nori, Phys. Rev. Lett. 98, 077002 (2007); S. Savel'ev, A. O. Sboychakov, A. L. Rakhmanov, and F. Nori, Phys. Rev. B 77, 014509 (2008); A. O. Sboychakov et al., Europhys. Lett. 80, 17009 (2007); A. O. Sboychakov, S. Savel'ev, and F. Nori, Phys. Rev. B 78, 134518 (2008).

[9] S. Coleman, Phys. Rev. D 15, 2929 (1977); A. O. Caldeira and A. J. Leggett, Ann. Phys. (N.Y.) 149, 374 (1983).

[10] When $T<T^{*}$, one can use a weaker inequality, $h \Omega \ll T^{*}$, since $B$ depends weakly on $T$ in this region. 